\title{
New autochthonous vines of Campania under evaluation for inclusion in productive sector
}

\author{
A.R. Caputo ${ }^{1}$, V. Alba ${ }^{1}$, C. Bergamini ${ }^{1}$, M. Gasparro ${ }^{1}$, G. Masi ${ }^{1}$, L. Tarricone ${ }^{1}$, P. Crupi $^{1}$, S. Roccotelli ${ }^{1}$, R. Pepe ${ }^{2}$ \\ e S. Del Lungo ${ }^{3}$ \\ ${ }^{1}$ Consiglio per la ricerca in agricoltura e l'analisi dell'economia agraria di ricerca, Centro di ricerca Viticoltura ed Enologia, \\ Via Casamassima, 148, 70010 Turi (BA), Italia \\ ${ }^{2}$ Consiglio per la ricerca in agricoltura e l'analisi dell'economia agraria, Centro di ricerca Orticoltura e Florovivaismo, \\ Via Cavalleggeri, 25, 84098 Pontecagnano (SA) Italia \\ ${ }^{3}$ Consiglio Nazionale delle Ricerche, Istituto per i Beni Archeologici e Monumentali, 85050 Tito Scalo (PZ), Contrada Loya, Italia
}

\begin{abstract}
The drastic reduction of agrobiodiversity has induced all countries in the world to implement numerous initiatives to safeguard genetic resources. The biodiversity issue is also one of the priority objectives of the global action plan for the conservation and sustainable use of FAO's cytogenetic resources and of the International Treaty on Plant Genetic Resources for Food and Agriculture. In Italy, the primary competence on genetic resources of agricultural interest is entrusted to the Ministry of Agricultural. In 2004, the Ministry entrusted the coordination of the RGV/FAO Project to CREA. The main objectives of the project, in addition to the inventory of accessions present in the collections of the structures involved, are their morphological, agronomic and molecular characterization and the productive enhancement with particular reference to the autochthonous varieties at risk of extinction. The project in question includes the activities of recovery and characterization of the viticultural germplasm illustrated in the present work. The 12 vines described here, like others yet to be rediscovered, are strongly linked to small winegrowers and indissolubly characterial of a territory in Campania Region that from the socio-cultural (rather fragmented agricultural system) and environmental orography point of view, like the national one, is very diversified.
\end{abstract}

\section{Introduzione}

Nell'ambito dell'agro-biodiversità italiana, il patrimonio viticolo autoctono, per la sua lunga storia dalla prima domesticazione alla moderna coltivazione, rappresenta uno degli elementi a più forte rinomanza per il territorio in cui si è insediato. A partire dall'anno 2004, in cui l'Italia ha ratificato l'adesione al Trattato Internazionale della FAO sulle Risorse fitogenetiche per l'alimentazione e l'agricoltura (ITPGRFA) [1], e fino al 2010, nel Mezzogiorno d'Italia, numerose sono state le iniziative intraprese per salvaguardare la biodiversità viticola.

\subsection{La riscoperta del germoplasma viticolo campano}

Fra le regioni meridionali, la Campania, nonostante la base varietale piuttosto ampia e localmente rappresentativa, conosce ancora poco del proprio patrimonio genetico viticolo.

Dopo un periodo di interruzione, è stato sufficiente riprendere nell'ultimo triennio l'attività di esplorazione per individuare altri nuclei di origine e diffusione di risorse genetiche inespresse, dalla punta estrema del Cilento (Policastro Bussentino), fino alle coste della penisola sorrentina (Gragnano e Vico Equense), con un passaggio nell'entroterra fra le colline beneventane di Solopaca e Castelvenere.

\subsection{Inquadramento storico geografico}

I profili molecolari delle varietà individuate, reinterpretati alla luce delle fonti storiche e del dato archeologico, testimoniano una condivisione di patrimonio genetico che richiede un approfondimento.

Rispetto alle apparenze non ci sono barriere culturali fra Golfo di Napoli ed entroterra sin dai tempi della colonizzazione magnogreca. Una circolazione la anticipano in età classica l'Aglianico n. e l'Aglianico bianco (vitigno autoctono caratterizzato recentemente e in corso di iscrizione al Registro Nazionale delle Varietà di Vite - RNVV, articolo 10 del Decreto ministeriale 8 febbraio 2005), in movimento dalla Basilicata al comprensorio Vesuviano fino al Beneventano e da lì di nuovo in Basilicata. Costa ed entroterra sviluppano una propria biodiversità viticola beneficiando dei contatti e degli scambi reciproci di varietà.

Ancora in pieno XVI secolo la Penisola Sorrentina costituisce con il Golfo di Napoli, il Vesuvio e il Beneventano un sistema di aree viticole fortemente vocate e in stretta relazione fra loro. Delineano il fenomeno la prossimità molecolare delle varietà indagate e le sinonimie introdotte ad uso commerciale (il Grecum vinum per la Cocozza b., la Agostina b. e 1'Uva Urmo b.; la Vernacia per le Vernacce d'Arulo e di Vigna, entrambe nere, vere e presunte; il Lachrima e il Mangiauerra per la Tentiglia n., il Sabato n., la Castagnara n. e la Suppezza n.; l'Aglianico, per la Reginella n.). Resta

(C) The Authors, published by EDP Sciences. This is an Open Access article distributed under the terms of the Creative Commons Attribution License 4.0 (http://creativecommons.org/licenses/by/4.0/). 
invece fortemente ancorato ai territori montani di origine l'Nganna pastore b., spostandosi come la Santa Sofia b. (altro vitigno autoctono caratterizzato recentemente e in corso di iscrizione al RNVV) in modo autonomo su percorsi interni appoggiati agli Appennini.

\section{Materiali e metodi}

Ogni entità varietale descritta nel lavoro è stata segnalata da viticoltori campani, autentici custodi di varietà locali.

Le prime tre varietà, nell'ordine come sono state citate nella sezione precedente, Cocozza, Agostina e Uva Urmo, sono state reperite in provincia di Benevento, nei comuni di Solopaca e Castelvenere, che ricadono nell'area della DOC "Sannio"; relativamente alla fase fenologica della maturazione, sono vitigni di seconda e terza epoca: "medio-precoce" la prima (terza decade di agosto-prima di settembre), "normale" o "media" la seconda e la terza (seconda-terza decade di settembre). Anche le due Vernacce d'Arulo e di Vigna sono originarie dell'area beneventana con la maturazione che avviene normalmente nella seconda decade di settembre, in terza epoca. Chiude il gruppo delle nuove varietà beneventane la Tentiglia, anch'essa di normale o media maturazione, a metà settembre.

I tre vitigni che seguono sono stati, invece, recuperati nella Penisola Sorrentina, in provincia di Napoli, tra il golfo di Napoli, i monti Lattari e il golfo di Salerno: il Sabato, che matura tardivamente a metà ottobre, nel comune di Vico Equense; la Castagnara e la Suppezza, più precoci del precedente, che maturano nella terza decade di settembre, nel comune di Gragnano. Seguono i due vitigni omonimi, le Reginelle, differenti tra loro per l'epoca di maturazione, ritrovati nel territorio di Policastro Bussentino, in provincia di Salerno (ai confini con la Basilicata): una Reginella (d'ora innanzi denominata precoce) matura in prima epoca (precoce ritardata) nella seconda metà di agosto, mentre, la seconda Reginella (denominata tardiva, per distinguerla dalla prima) matura in seconda epoca, medio-precoce, verso la fine di agosto. Infine, chiude l'elenco delle varietà oggetto di osservazione, l'Nganna pastore, un vitigno che matura nella prima metà di ottobre, recuperato ad Alvignano, in provincia di Caserta, in un territorio limitrofo alle colline beneventane.

\subsection{Caratterizzazione varietale}

La caratterizzazione dei nuovi ritrovati vegetali che di seguito viene illustrata è stata effettuata sulle piante madri originarie o fonti primarie direttamente nei siti di ritrovamento.

\subsubsection{Determinazione dei profili genetici}

L'identificazione molecolare è stata effettuata mediante l'estrazione del DNA genomico (DNAeasy®Plant Mini Kit, Qiagen, Hilden Germany) e l'amplificazione mediante PCR (Polymerase Chain Reaction) di marcatori molecolari SSR di-nucleotidici: i sei definiti dal progetto Genres CT96 No81 (European Vitis Database, www.genres.de/vitis/vitis.htm) e altri tre individuati in letteratura come altamente polimorfici e riproducibili. I frammenti amplificati nelle reazioni di PCR sono stati analizzati con un sequenziatore ad 8 capillari (CEQ8000, Beckman Coulter Inc., CA USA).

Sono stati impiegati, quindi, 9 diversi marcatori SSR, selezionati per la possibilità di essere confrontati con il database presente nel Registro Nazionale delle Varietà di Vite (RNVV) del Ministero delle Politiche Agricole Alimentari Forestali e del Turismo (Mi.P.A.A.F.T., http://catalogoviti.politicheagricole.it/ catalogo.php) e con i principali database di riferimento: il catalogo europeo "Vitis International Variety Catalogue" (VIVC, http: //www.vivc.de) e l'altro catalogo nazionale http://www.vitisdb.it, oltre naturalmente al database interno del CREA-VE di Turi (BA), costituito quest'ultimo da quasi un migliaio di diversi profili unici di varietà di uve da vino e da tavola di provenienza internazionale, nazionale e in particolare di vitigni autoctoni del meridione d'Italia. L'analisi è stata condotta sui seguenti 9 SSR: VVS2, VVMD5, VVMD7, VVMD27, VrZAG62, VrZAG79 [2], VVMD25, VVMD28 e VVMD32 [3].

\subsubsection{Descrizione morfologica e produttiva}

I vitigni identificati - inoltre - sono stati caratterizzati mediante descrittori ampelografici primari e secondari rilevati su germoglio giovane, foglia giovane e adulta, grappolo e acino, utilizzando la metodica della II $^{\mathrm{a}}$ Edizione del Codice dei caratteri descrittori dell'O.I.V. (Scheda ampelografica, 2001); a carico del grappolo e degli acini sono stati rilevati parametri quanti-qualitativi (peso medio grappolo, peso medio acino, zuccheri, $\mathrm{pH}$ e acidità totale) e produttivi (fertilità potenziale, intesa come numero di grappoli per numero di gemme germogliate), oltre al peso del legno di potatura (sarmenti); infine, con misurazioni ampelometriche delle nervature principali e degli angoli da esse delimitate, effettuate con il software "SuperAmpelo", è stata ricostruita l'immagine della foglia standard.

\section{Risultati}

Nella Tabella 1 si riportano i profili molecolari unici dei nuovi vitigni autoctoni individuati con le denominazioni in vernacolo. I profili genetici sono rappresentati con caratteri descrittivi (marcatori SSR), espressi in valori pb (paia di basi) osservati nella coppia di alleli di ciascun microsatellite, indicati come distanze relative espresse in paia di basi dall'allele di riferimento di dimensione $\mathrm{n}$.

Per quanto riguarda la caratterizzazione fenotipica, a titolo semplificativo, è stata presa in considerazione la "lista prioritaria dei descrittori primari": OIV-001, Giovane germoglio: apertura dell' apice; OIV-004, Giovane germoglio: densità di peli striscianti dell'apice; OIV-016 Germoglio: numero di viticci consecutivi; OIV-051, Foglia giovane: colore della pagina superiore del lembo $\left(4^{\mathrm{a}}\right.$ foglia); OIV-067, Foglia adulta: forma del lembo; OIV068, Foglia adulta: numero dei lobi; OIV-070, Foglia adulta: distribuzione della pigmentazione antocianica sulle nervature principali della pagina superiore del lembo; OIV-076, Foglia adulta: forma dei denti; OIV079, Foglia adulta: grado di apertura/sovrapposizione del seno peziolare; OIV-081/2, Foglia adulta: base del seno peziolare delimitata dalle nervature; OIV-084, Foglia adulta: densità dei peli striscianti tra le nervature principali sulla pagina inferiore del lembo; OIV-087, Foglia 
Tabella 1. Profili molecolari dei vitigni autoctoni.

\begin{tabular}{|cccccccccc|} 
Varietà & VVS2 & MD5 & MD7 & MD25 & MD27 & MD28 & MD32 & ZAG62 & ZAG79 \\
Cocozza b. & 20 & 4 & 16 & 6 & 4 & 32 & 23 & 20 & 6 \\
& 22 & 18 & 16 & 20 & 4 & 42 & 37 & 22 & 20 \\
Agostina b. & 12 & 4 & 16 & 6 & 6 & 12 & 23 & 16 & 22 \\
& 22 & 6 & 18 & 20 & 6 & 32 & 37 & 20 & 22 \\
Uva Urmo b. & 10 & 4 & 8 & 6 & 4 & 32 & 27 & 16 & 6 \\
Vernaccia & 14 & 18 & 16 & 6 & 8 & 42 & 37 & 22 & 10 \\
d'Arulo n. & 34 & 10 & 12 & 4 & 10 & 20 & 15 & 14 & 14 \\
Vernaccia di & 22 & 6 & 32 & 6 & 14 & 42 & 21 & 20 & 22 \\
Vigna n. & 22 & 10 & 18 & 6 & 4 & 28 & 17 & 14 & 14 \\
Tentiglia n. & 20 & 16 & 8 & 4 & 10 & 44 & 37 & 16 & 22 \\
& 32 & 24 & 32 & 14 & 14 & 42 & 21 & 20 & 8 \\
& 28 & 14 & 8 & 6 & 4 & 18 & 15 & 14 & 6 \\
Sabato n. & 32 & 16 & 12 & 14 & 16 & 42 & 17 & 22 & 18 \\
Castagnara n. & 10 & 10 & 32 & 4 & 10 & 42 & 21 & 20 & 14 \\
& 28 & 16 & 32 & 6 & 10 & 42 & 23 & 22 & 14 \\
Suppezza n. & 10 & 12 & 8 & 14 & 10 & 18 & 17 & 14 & 14 \\
Reginella & 28 & 18 & 8 & 28 & 10 & 42 & 21 & 22 & 14 \\
precoce n. & 32 & 10 & 8 & 6 & 4 & 12 & 21 & 22 & 14 \\
Reginella & 28 & 4 & 8 & 14 & 4 & 12 & 17 & 14 & 8 \\
tardiva n. & 32 & 10 & 18 & 20 & 14 & 12 & 21 & 28 & 14 \\
Nganna & 10 & 4 & 8 & 4 & 14 & 12 & 17 & 14 & 6 \\
pastore b. & 28 & 14 & 16 & 28 & 14 & 32 & 19 & 20 & 22 \\
\hline
\end{tabular}

Tabella 2. Legenda dei livelli di espressione dei descrittori utilizzati.

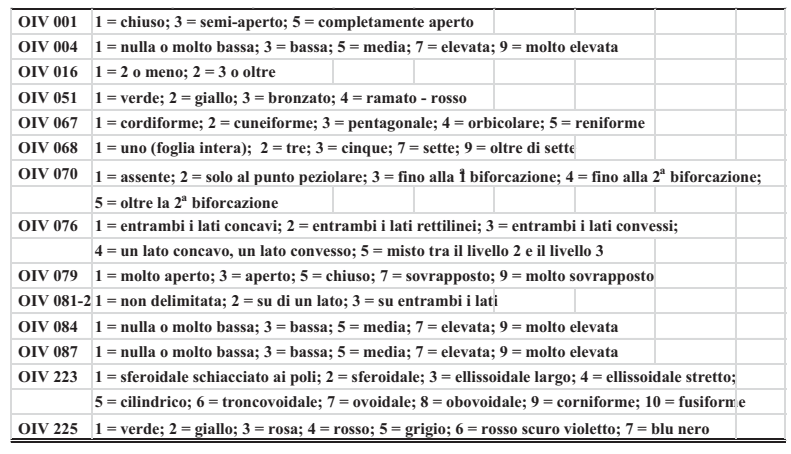

Tabella 3. Descrizione delle varietà mediante i 14 descrittori primari.

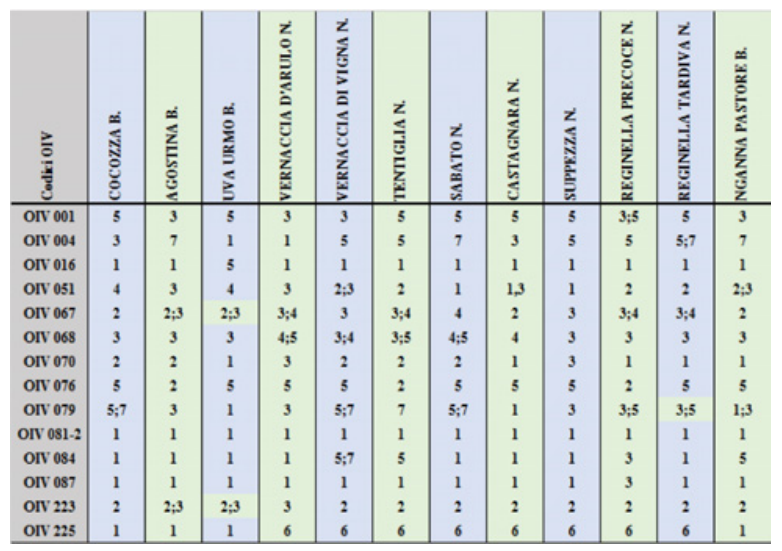

adulta: densità dei peli eretti sulle nervature principali della pagina inferiore; OIV-223, Acino: forma; OIV-225, Acino: colore della buccia. I livelli di espressione dei descrittori sono indicati nella seguente Tabella 2.

Per una lettura di comparazione immediata fra le varietà, di seguito si riportano i livelli di espressione dei caratteri ampelografici primari riscontrati (Tabella 3).

La Tabella 4, riporta i principali parametri produttivi ed eno-chimici del mosto, rilevati nel corso dell'ultimo ciclo vegeto-produttivo.
Tabella 4. Parametri quanti-qualitativi (2018).
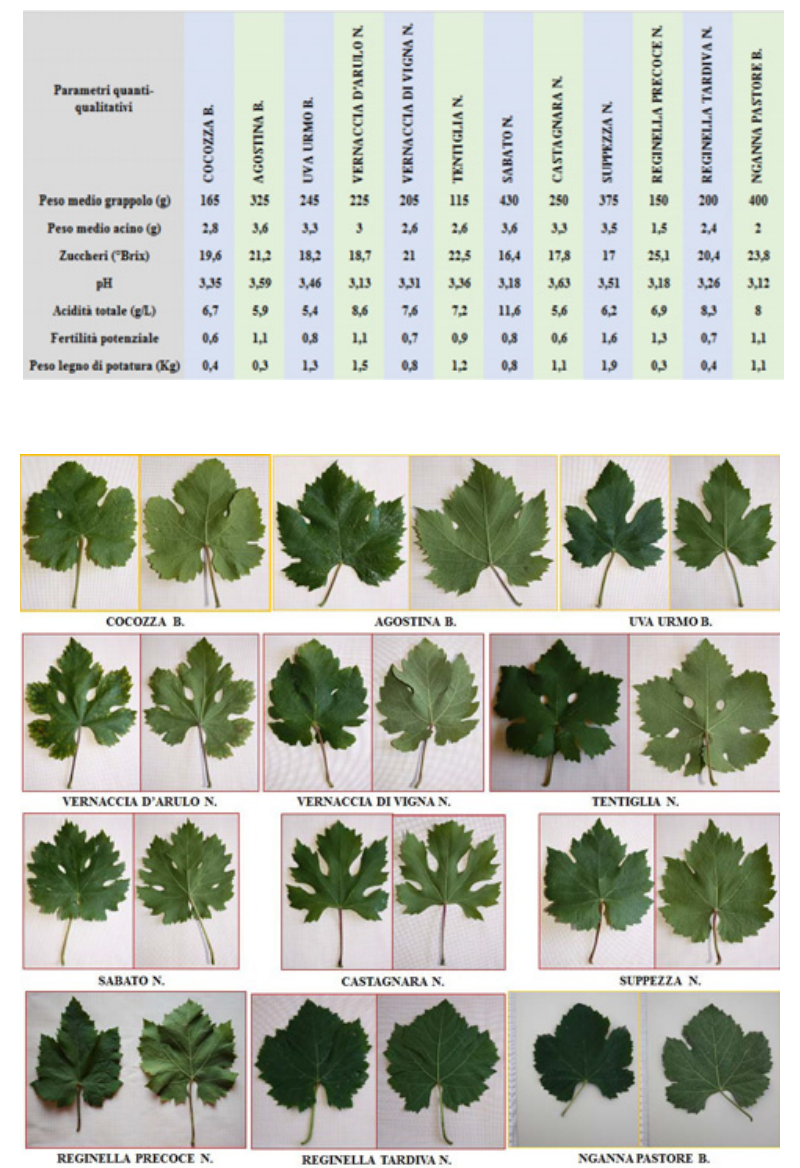

Figura 1. Foglie adulte.

Nelle figure che seguono sono messi a confronto gli organi osservati: foglie adulte, grappoli e immagini delle foglie standard.

\section{Considerazioni conclusive}

La piattaforma ampelografica italiana, come dimostrano le oltre 500 varietà ad uva da vino iscritte al Registro nazionale, è caratterizzata da una larga maggioranza di vitigni tradizionalmente coltivati nel nostro paese.

Tuttavia, alla luce dei risultati di questo lavoro, sembra chiara emergere la consapevolezza che diverse altre, coltivate e non, sono assenti dal già ricco elenco di varietà locali minori. Sull'importanza strategica della diversità varietale, e in particolar modo di quella autoctona, basta richiamare il recente "Testo Unico del vino", la legge 12 dicembre 2016, n. 238, che ha riconosciuto come patrimonio culturale nazionale "il vino, prodotto della vite, la vite e $i$ territori viticoli, quali il frutto del lavoro, dell'insieme delle competenze, delle conoscenze, delle pratiche e delle tradizioni..." e ha, contestualmente introdotto una definizione innovativa di "vitigno autoctono italiano", in quanto nativo di aree delimitate del territorio viticolo, e i vigneti eroici o storici, di particolare interesse paesaggistico, storico o ambientale. È certamente un profondo cambiamento di approccio che vede nella valorizzazione del territorio viticolo e nei vitigni autoctoni da preservare elementi identitari non solo storico-culturali ma anche strumenti concreti per 


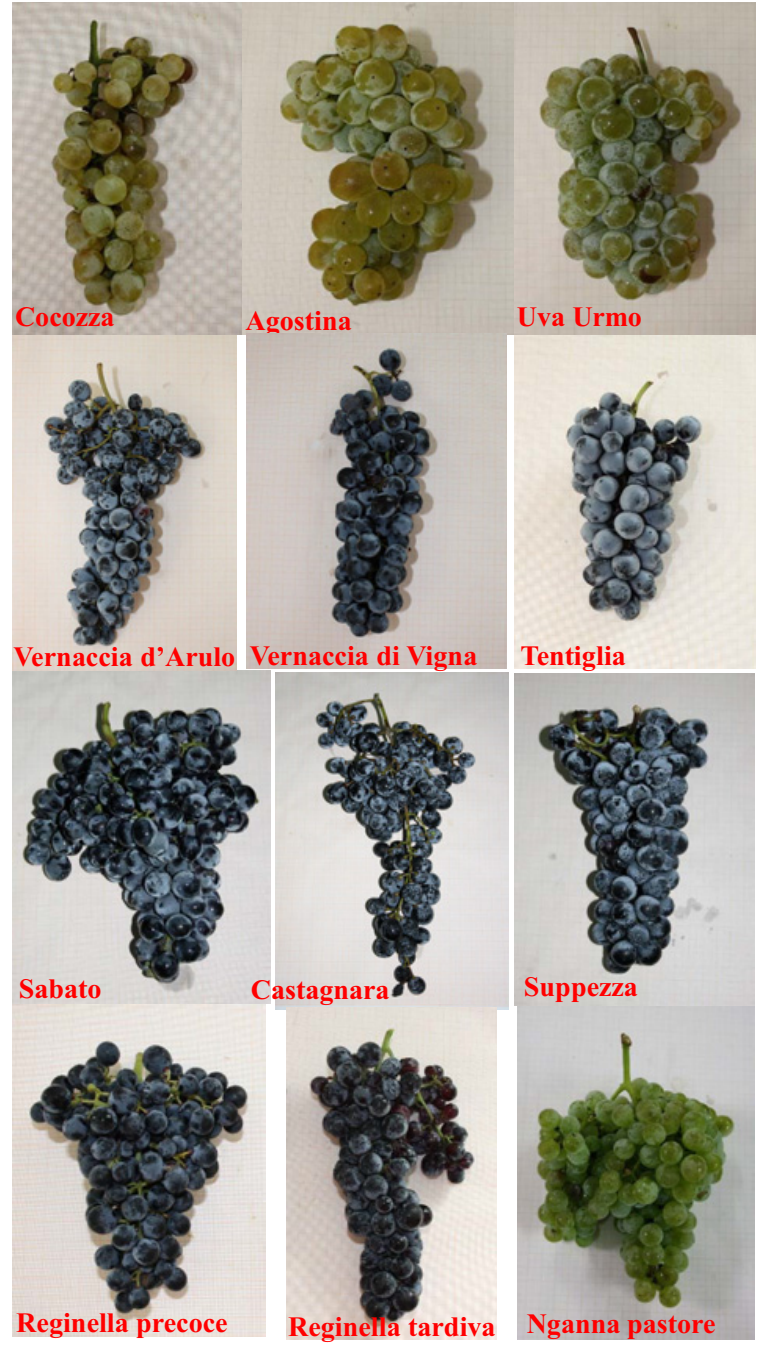

Figura 2. Grappoli a maturazione.

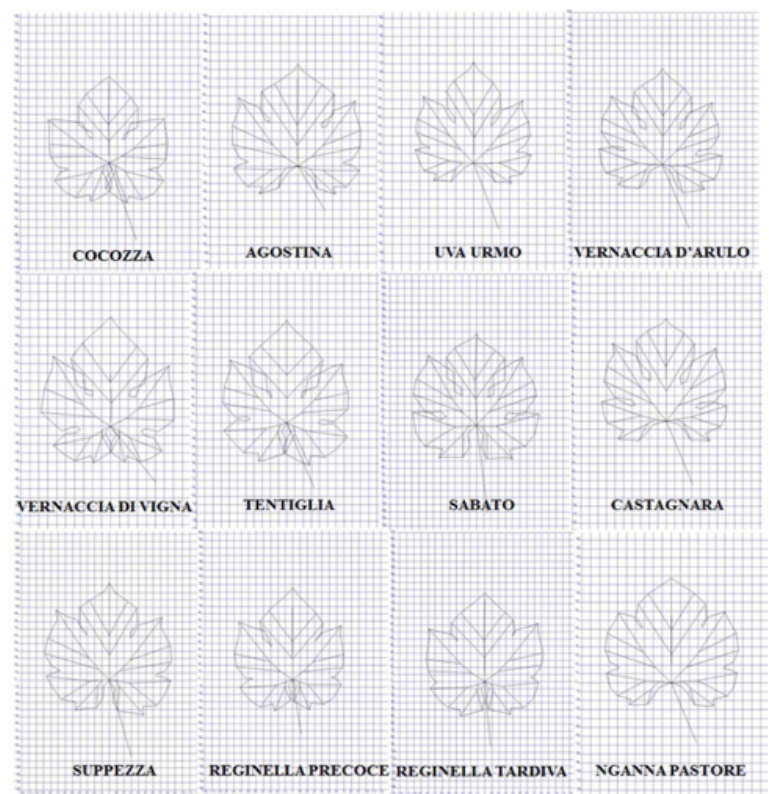

Figura 3. Ricostruzione delle foglie medie standard. accrescere lo sviluppo imprenditoriale grazie ad un'offerta commerciale di vini più ampia e differenziata ottenuti da vitigni inopportunamente dimenticati.

In tale contesto, la ricerca sia pubblica che privata, deve pertanto assumersi la responsabilità di assicurare il ricco patrimonio ampelografico ancora da scoprire, di valorizzarlo, perché sia il più possibile reintrodotto.

Naturalmente, questa operazione deve avvenire attraverso una rigorosa selezione e individuazione di vitigni fra quelli più validi dal punto di vista produttivo ed enologico per un loro potenziale utilizzo commerciale.

I vitigni oggetto di studio del presente lavoro, pur essendo stati esaminati solamente mediante parametri primari, hanno comunque evidenziato delle potenzialità importanti, come ad esempio la precocità di maturazione del vitigno Reginella, che presenta pure grappoli con acini di piccolissime dimensioni $(1.5 \mathrm{~g})$; stesse caratteristiche che ritroviamo nella Tentiglia, con un peso grappolo di poco superiore a $100 \mathrm{~g}$ e acini con una colorazione molto intensa (blu nero), molto ricca in antociani. Altrettanto interessanti per produttività sono risultate le due Vernacce (d'Arulo e di Vigna), il Sabato e la Suppezza; chiude l'elenco, l'Nganna pastore, un vitigno bianco mediamente produttivo con peso medio grappolo intorno a $400 \mathrm{~g}$ e con caratteristiche qualitative di particolare interesse, soprattutto per il suo tenore di acidità.

Quanto su esposto è il risultato di una prima indagine preliminare, che permette però di candidare i vitigni "in osservazione" per un potenziale inserimento nella piattaforma ampelografica regionale. Prima di una effettiva reintroduzione, però, gli approfondimenti continuano con lo studio dell'attitudine alla coltura di viti ottenute per moltiplicazione vegetativa delle piante originarie.

\section{Ringraziamenti}

I ringraziamenti vanno a coloro che hanno contribuito al lavoro di recupero: a tutti i viticoltori custodi che ci hanno consentito di visitare i loro vigneti o le loro vigne storiche; ai funzionari della Regione Campania, Alberto Ziello e Carlo Sardo, che ci hanno fatto da guida nel lavoro di esplorazione del territorio. Inoltre, si ringrazia la collega, Sig.ra Teresa Nobile, per il lavoro di supporto nell'implementazione dei dati per la preparazione dei fascicoli tecnico-amministrativi che saranno utilizzati per il riconoscimento ufficiale delle varietà oggetto di studio.

\section{Riferimenti}

[1] A.A.V.V. Linee Guida per La Conservazione e La Caratterizzazione Della Biodiversità Vegetale di Interesse per L'agricoltura (Mi.P.A.A.F., INEA, Roma, 2013)

[2] P. This, A. Jung, P. Boccacci, J. Borrego, R. Botta, L. Costantini, M. Crespan, G.S. Dangl, C. Eisenheld, F. Ferreira-Monteiro, M.S. Grando, J. Ibanez, T. Lacombe, V. Laucou, M. Magalhaes, C.P. Meredith, N. Milani, E. Peterlunger, F. Regner, L. Zulini, E. Maul, Theor. Appl. Gen. 109, 1448 (2004)

[3] J.E. Bowers, G.S. Dangl, C.P. Meredith, Am. J. Enol. Vitic. 50, 243 (1999) 